\title{
Enhancement of Life-threatening Arrhythmia Discrimination in the Intensive Care Unit with Morphological Features and Interval Feature Extraction via Random Forest Classifier
}

\author{
Farhad Asadi $^{1}$, Mohammad Javad Mollakazemi ${ }^{1,2}$, Shadi Ghiasi ${ }^{1,2}$, S. Hossein Sadati ${ }^{1}$ \\ ${ }^{1}$ Department of Mechanical Engineering at K N Toosi University of Technology, Tehran, Iran \\ ${ }^{2}$ Cardiovascular Research Group (CVRG), Department of Mechanical Engineering, \\ K N Toosi University of Technology, Tehran, Iran
}

\begin{abstract}
Introduction: Intelligent patient monitoring has continued to enhance and develop in hospitals from the early stage of monitoring systems. So, practical medical monitoring devices to react to patient conditions and also detect unwanted clinical conditions are very important.

Aims: Our algorithm uses pulsatile waveforms and simultaneous ECG in order to detect and enhance the determination of the life-threatening arrhythmia alarms in the context of the PhysioNet/Computing in Cardiology 2015 Challenge.

Methods: the analysis steps included: In our algorithm, features for training the random forest classifier (RFC) were derived from applying the signal quality assessment (SQI) to both pulsatile signals and ECG signal too. Primarily, preprocessing step was done by applying the band pass filters to multiple sources, such as arterial blood pressure $(A B P)$, photoplethysmogram (PPG) and electrocardiogram (ECG) and then heart beat detection through the adaptive threshold were determined. The SQI approach for the pulsatile signals was applied through the ppgSQI and the jSQI algorithms and also spectral and statistical features were extracted from ECG channel as well. In a next process, the heuristic thresholding of each $A B P$ pulse are estimated with the function of abpfeature and also heart rate (HR) features from the ECG and pulsatile signals in a segment before the alarm was extracted and computed. Also, for assessing regularity of the beats, inter-beat intervals for pulsatile waveforms and also checking the frequency maxims for better suppression of ventricular flutter/fibrillation in the ECG channel were computed. Finally, RFCs were trained with arrhythmia features set for every type of the arrhythmia.

Results: our algorithm was trained with the use of 750 records provided by PhysioNet dataset for the challenge of 2015 and according to the types of arrhythmia, our overall scores varied. Our average score for our best performance for all the alarms in terms of true positive were $67 \%$ and for true negative were $77 \%$ and for false negative were $1.8 \%$.
\end{abstract}

1.

Introduction

Arrhythmias are abnormalities in heart function which some of them are greatly dangerous or even lifethreatening in healthy individuals. Intensive care units (ICU) are facilitated to identify life-threatening arrhythmias as ventricular flutter/fibrillation (oscillatory waveform for more than $4 \mathrm{~s}$ ), bradycardia (HR $<40$ bpm), tachycardia (HR $>140$ bpm), ventricular tachycardia (a sequence of ventricle beats at $\mathrm{HR}>100$ bpm) and asystole (missing beats for more than $4 \mathrm{~s}$ ). The existence of false alarms during the Intensive Care Units (ICU) cause a delay in reaction time or decrease the chance of caregivers to appropriate responding or enhancement of the quality of patient care since the sensitivity of algorithm are intentionally set to high [1]. Furthermore, the source of these alarms could be varied corresponding to the unintended noises ascending from motion artifacts, muscle contractions, temporary machine faults such as detachment of electrodes and sensors or algorithm performance [2, 3]. In the ICU, the average of false arrhythmia can be increased by the value of $88.8 \%$ but different physiological signals which contain balancing information are used by clinicians to recognize these alarms [4]. Therefore, a robust approach to HR calculation with employing accurate signal quality index (SQI) can be utilized to reduce the false alarms and enhance the ICU monitoring. Moreover, although, if the whole range of noises can be taken into consideration the false alarm ratio might increase to amount of $86 \%$ but, in this improvement, it is not suitable to miss any lifethreatening arrhythmias and whenever anything doubtful occurs, the algorithm must be identify the type and also detect the alarm in order to save a life [5]. The task set by the Physionet "CinC Challenge 2015" was to create an algorithm to decline the false alarm ratio of 5 lifethreatening arrhythmias. If a given ICU alarm does not match into one of the 5 alarm types of data, the alarm will be categorized as a false alarm [6]. Since the quality and features of the different waveforms are a major issue in most cases, the ability of the algorithm to enhance the 
signal quality indexes (SQIs) for signals especially for pulsatile signals, can contribute to the development of the decision-making process $[7,8]$. Moreover, the algorithm should be able to assign different SQIs criteria with reasonable and accurate thresholds which can intensely discriminate the valuable characteristic of each arrhythmia with noises or others artifacts and then classify these features accurately $[9,10]$. Moreover in the CinC Challenge 2015, for enhancing the decision-making process, some machine learning approaches such random forest classifier or morphological features are used [11, 12]. The principal of our approach relies on the accurate and reliable detection of the heartbeats features and also features from both the pulsatile and ECG signals. This study aims to reduce the false alarms (FAs) in the ICU and also our algorithm relies on robust adaptive signal processing techniques in order to extract different reliable features and $\mathrm{HR}$ values from the ECG, photoplethysmogram (PPG) and ABP waveforms.

\section{Material and methods}

\subsection{Data preparation}

The training dataset for the proposed algorithm is a 5minute-long record, 750 records, from the ICU monitor at $250 \mathrm{~Hz}$ which it was provided by the PhysioNet/Computing in Cardiology Challenge 2015. Each record contained an alarm which was either true or false for one arrhythmia occurrence and also each recording contained two leads of ECG, and at least two pressure channels with arterial blood pressure (ABP) and/or photoplethysmograph (PPG). Firstly, the overall structure of the proposed algorithm is plotted in fig.1 which consists of three steps of quality assessment step, feature extraction, and finally decision-making process. Then, detailed steps of the algorithm are explained in below sections.

\subsection{Quality assessments for pulsatile and ECG signal}

In order to detect the pulse from the ABP and PPG signals, we used the open-source $\mathrm{ABP}$ pulse detection algorithm which is available in PhysioNet [6]. Since, ECG channels in the training set showed that the available ECG signals existing various disturbances such as clipping of the QRS complexes, and other noises, beats detected should be accomplished from different channels and signals and then compared them with each other. So, the best ECG channel or ABP and PPG waveforms after signal quality assessments for them are chosen for feature extraction. It is noticeable to say that delays between ECG and ABP and PPG are computed and considered in the algorithm. Furthermore, the ppgSQI and the jSQI algorithms which are provided for the PhysioNet/Computing in Cardiology 2015 Challenge are used for assessment of PPG and ABP signal. These algorithms are based on a beat template correlation which is shaped by averaging the detected beats from the signal. The evaluation of the signal quality of ABP signal, ABP SQI calculation was based on a beat-by-beat level assessment of features in the ABP waveform. This index which is known as jSQI, extract several features from the signal and give a flag ' 1 ' if the feature does not encounter the criterion of normality. Also, in the algorithm, the feature flags were changed to a quality index between 0 and 1 that 1 correspond to a clean signal respectively. Next, an approach from the reference $[11,13]$ is used for ECG R-peak detection algorithm which is optimized to decrease computational complexity. In this algorithm, firstly, a signal segmentation is applied in each iteration of the algorithm and then adaptive threshold determination, peak detection and finally SNR estimation are employed in order to search the peak of the signal.

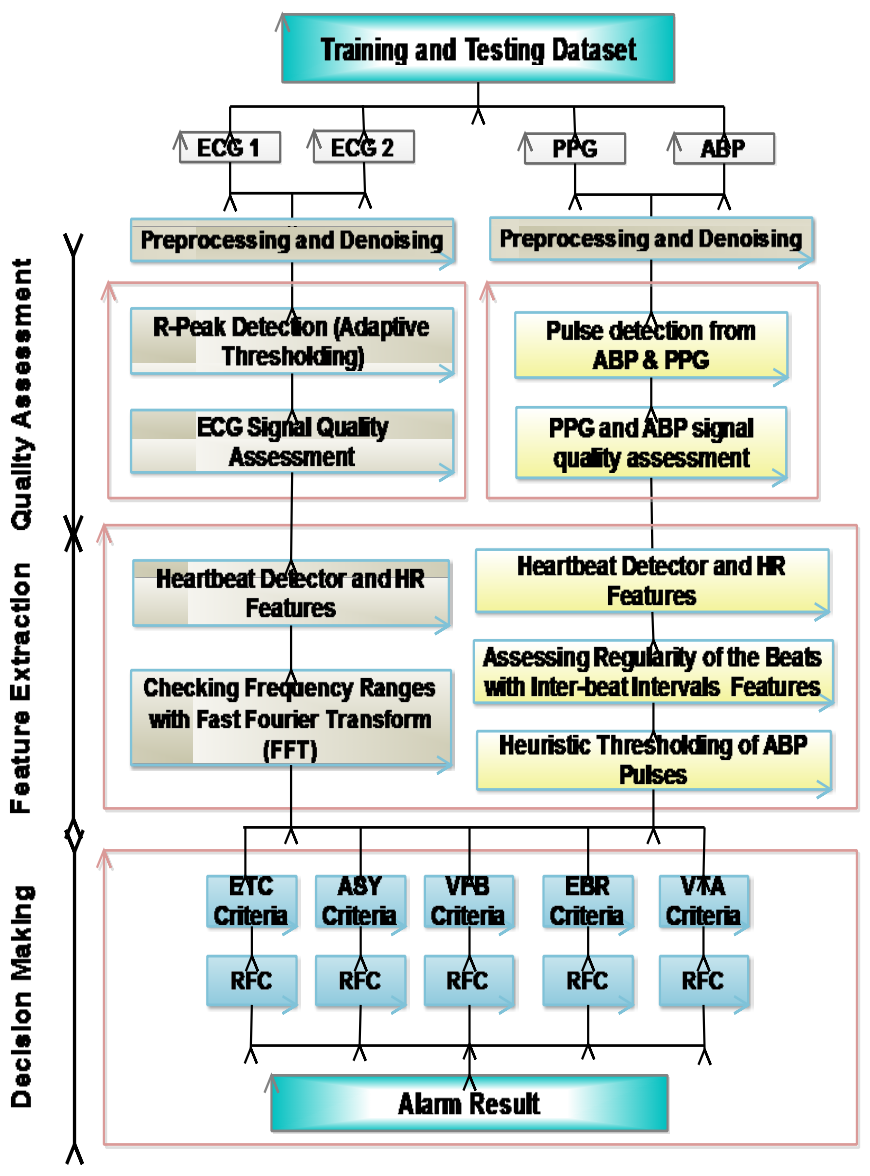

Figure. 1 The overall block diagram of approach 


\subsection{Heart beat detection and feature extraction}

In this section of algorithm, we focused on 5 life threatening arrhythmias, namely extreme tachycardia (ETC), ventricular fibrillation or flutter (VFB), asystole (ASY), extreme bradycardia (EBR), or ventricular tachycardia (VTA) and based on the clinical definition and different characteristics of each of them, signal quality features are used for them [6]. In our algorithm, the heuristic thresholding of each ABP pulse are computed with the function of abpfeature, and also, since blood pressure variation can occur during asystole and ventricular tachycardia, these features can detect these abnormalities accurately.

By using this function, different features from $\mathrm{ABP}$ waveform such as systolic pressure, mean pressure and others can be extracted and used for each arrhythmia accordingly. Next, heart rate (HR) features from the ECG and pulsatile signals in a segment before the alarm was extracted and computed. Moreover, for better suppression of ventricular fibrillation or flutter which can be characterized as continuous low-frequency activity distinctively, ECG channels are investigated for 2 secondwindow for checking frequency maxims with Fast Fourier Transform (FFT).

Generally, ventricular fibrillation epoch is distinctive from other arrhythmias types for its amplitude in the frequency range above the $2 \mathrm{HZ}$. Furthermore, for assessing and evaluating the regularity of the beats, interbeat intervals is computed and its deviation for pulsatile signals are computed and used for some of the arrhythmias.

So, these computed features after computing from the signals are ready to assign to each of arrhythmias based on defined and supposed characteristics for them. In the algorithm, each of the arrhythmias is processes separately and details about waveforms, the extracted features for them are shown in Table 1. So, these computed and obtained features are ready to assign to each of arrhythmias based on defined and supposed characteristics for them. In the algorithm, each of the arrhythmias criteria is defined separately and details about waveforms, the extracted features for them are shown in Table 1.

\subsection{The judgment of false alarms with random forest classifier}

It is noticeable to say that after applying the SQIs approaches to ECG and pulsatile signals, the mentioned features for arrhythmias were computed and the defined criterion for each arrhythmia was calculated for the classification. The random forest classifier which is relatively robust to noisy data is used and applied for classification between true and false alarms with a separate random forest classifier for every alarm.

\section{Results}

Based on the method describe above, false alarm classification in the training set (750 records) and the test set of PhysioNet database were evaluated. About half of the records were used for training and another half of the data were used for validation. According to the challenge, the performance of the algorithm was rated with true positive rate (TPR) and true negative rate (TNR) and false negative rate (FNR) within the datasets. The outcome results for the five type of arrhythmias in both of the training and validating dataset were listed in Table 2 and Table 3 as well.

Table 1. Features for classification of Arrhythmia

\begin{tabular}{cccc}
\hline $\begin{array}{c}\text { Arrhythmia } \\
\text { type }\end{array}$ & Waveforms & $\begin{array}{c}\text { Algorithms for the } \\
\text { computing SQI }\end{array}$ & Extracted Features for each arrhythmias \\
\hline ETC & ECG & $\begin{array}{c}\text { Adaptive thresholding } \\
\text { jSQI, ppgSQI }\end{array}$ & $\begin{array}{c}\text { Systolic blood pressure (SBP), maximum HR of 13 } \\
\text { consecutive beats, maximum number of consecutive } \\
\text { beats with HR under 140 bpm }\end{array}$ \\
\hline ASY & ABP/PPG & jSQI, ppgSQI & $\begin{array}{c}\text { Maximum inter-beat intervals, diastolic blood } \\
\text { pressure (DBP) and mean blood pressure (MBP) }\end{array}$ \\
\hline VFB & ECG & $\begin{array}{c}\text { Adaptive thresholding } \\
\text { jSQI, ppgSQI }\end{array}$ & $\begin{array}{c}\text { Frequency maxims criteria in ECG signal, deviation } \\
\text { and amplitude of inter-beat intervals }\end{array}$ \\
\hline EBR & ECG & Adaptive thresholding & $\begin{array}{c}\text { Minimum HR of 6 consecutive beats- mean blood } \\
\text { pressure (MBP), maximum number of consecutive } \\
\text { beats with HR under 40 bpm }\end{array}$ \\
\hline jTA & ABP/PPG & ECG & Adaptive thresholding \\
jSQI, ppgSQI & $\begin{array}{c}\text { Maximum HR, range of pulse pressure, diastolic } \\
\text { blood pressure, systolic blood pressure, inter-beat } \\
\text { intervals }\end{array}$ \\
\hline
\end{tabular}


Table 2. Classification results for training dataset

\begin{tabular}{cccc}
\hline $\begin{array}{c}\text { Arrhythmia } \\
\text { type }\end{array}$ & $\begin{array}{c}\text { TPR } \\
(\mathbf{\% )}\end{array}$ & $\begin{array}{c}\text { TNR } \\
(\mathbf{\% )}\end{array}$ & $\begin{array}{c}\text { FNR } \\
\mathbf{( \% )}\end{array}$ \\
\hline ETC & 0.87 & 0.82 & 0.023 \\
ASY & 0.39 & 0.49 & 0.011 \\
VFB & 0.78 & 0.89 & 0.018 \\
EBR & 0.53 & 0.6 & 0.019 \\
VTA & 0.68 & 0.85 & 0.02 \\
Average & 0.65 & 0.73 & 0.018 \\
\hline
\end{tabular}

Table 3. Classification results for validation dataset

\begin{tabular}{cccc}
\hline $\begin{array}{c}\text { Arrhythmia } \\
\text { type }\end{array}$ & $\begin{array}{c}\text { TPR } \\
(\mathbf{\% )}\end{array}$ & $\begin{array}{c}\text { TNR } \\
\text { (\%) }\end{array}$ & $\begin{array}{c}\text { FNR } \\
\text { (\%) }\end{array}$ \\
\hline ETC & 0.89 & 0.9 & 0.02 \\
ASY & 0.38 & 0.51 & 0.013 \\
VFB & 0.81 & 0.91 & 0.018 \\
EBR & 0.53 & 0.64 & 0.021 \\
VTA & 0.72 & 0.89 & 0.02 \\
Average & 0.67 & 0.77 & 0.018 \\
\hline
\end{tabular}

\section{Discussion and conclusion}

After preprocessing of signals, we applied several signal quality assessments to remove and identify the effects of noise and others artifacts in order to enhance the beat detection and HR estimation and its features accordingly. It was found that applying the adaptive threshold approach for beat detection and suitable choice of signal quality threshold were crucial for reliable and robust estimating of HR estimation and can be caused by decreasing the occurrences of false alarm rate. For analyzing the VFB arrhythmias, we relied mostly on the ECG signal rather than on the pulsatile signals, since applying the frequency maxims criterion can be intensely displayed the presence of this arrhythmias according to the literature. Moreover, the role of parameters such as systolic blood pressure (SBP), diastolic blood pressure (DBP) and mean blood pressure (MBP) can be improved the alarm classification greatly since these parameters can be discerned and identified some of the arrhythmias with reasonable accuracy. Also, as a trial and error experiment in our algorithm, we found that the most distinctive feature for a false alarm can be identified as a detection of the high average rhythmicity of the signal and also deviation and amplitude of inter-beat interval for indicating a true alarm. Our work suggests that further improvement can be done by incorporating the fuzzy logic or machine learning methods in order to increase the accuracy of decision- making the process for assigning physiologically interpretable thresholds on the best features, which are obtained from the pulsatile waveforms.

\section{References}

[1] Overdyk FJ, Carter R, Maddox RR, Callura J, Herrin AE, Henriquez C. Continuous oximetry/capnometry monitoring reveals frequent desaturation and bradypnea during patientcontrolled analgesia. Anesthesia \& Analgesia 2007; 105(2):412-8.

[2] Görges M, Markewitz BA, Westenskow DR. Improving alarm performance in the medical intensive care unit using delays and clinical context. Anesthesia \& Analgesia 2009; 108(5):1546-52.

[3] Graham KC, Cvach M. Monitor alarm fatigue: standardizing use of physiological monitors and decreasing nuisance alarms. American Journal of Critical Care 2010; 19(1):28-34.

[4] Drew BJ, Harris P, Zègre-Hemsey JK, Mammone T, Schindler D, Salas-Boni R, Bai Y, Tinoco A, Ding Q, Hu $\mathrm{X}$. Insights into the problem of alarm fatigue with physiologic monitor devices: a comprehensive observational study of consecutive intensive care unit patients. PloS one. 2014; 9(10):e110274.

[5] Lawless ST. Crying wolf: false alarms in a pediatric intensive care unit. Critical care medicine. 1994; 22(6):9815.

[6] Clifford GD, Silva I, Moody B, Li Q, Kella D, Shahin A, Kooistra T, Perry D, Mark RG. The PhysioNet/computing in cardiology challenge 2015: reducing false arrhythmia alarms in the ICU. Computing in Cardiology 2015; 42:273276.

[7] Mollakazemi MJ, Atyabi SA, Ghaffari A. Heart beat detection using a multimodal data coupling method. Physiological measurement 2015; 36(8):1729.

[8] Asadi F, Mollakazemi MJ, Uzelac IL, Moosavian SA. A novel method for arterial blood pressure pulse detection based on a new coupling strategy and discrete wavelet transform. Computing in Cardiology 2015;42:1081-84.

[9] Li Q, Clifford GD. Suppress false Arrhythmia alarms of ICU monitors using heart rate estimation based on combined arterial blood pressure and ECG analysis. In2008 2nd International Conference on Bioinformatics and Biomedical Engineering 2008; 2185-2187.

[10] Asadi F, Mollakazemi MJ, Atyabi SA, Uzelac IL, Ghaffari A. Cardiac arrhythmia recognition with robust discrete wavelet-based and geometrical feature extraction via classifiers of SVM and MLP-BP and PNN neural networks. Computing in Cardiology 2015; 42:933-936.

[11] Eerik LM, Vanschoren J, Rooijakkers MJ, Vullings R, Aarts RM. Decreasing the false alarm rate of arrhythmias in intensive care using a machine learning approach. Computing in Cardiology 2015;42:293-296.

[12] Teo SK, Wong JC, Yang B, Yang F, Feng L, Lim TW, Su Y. Reducing false arrhythmia alarms in the ICU. In2015 Computing in Cardiology 2015;42:1177-1180.

[13] Rooijakkers MJ, Rabotti C, Oei SG, Mischi M. Lowcomplexity R-peak detection for ambulatory fetal monitoring. Physiological measurement 2012;33:1135.

Address for correspondence:

Farhad Asadi, Farhadasadi1990@gmail.com

K.N. Toosi University of Technology, No. 15, Pardis Street, MolaSadra Avenue, Vanak Sq.,Tehran, Iran. 